\title{
RAZONAR Y ESPECULAR
}

\author{
ENRIC TRILLAS \\ bttps://orcid.org/0000-0002-7245-7881 \\ Universidad Politécnica de Madrid \\ https://doi.org/10.15304/ag.40.1.6449
}

\section{Resumen}

Basadas en un modelo matemático muy simple del razonamiento de sentido común, se presentan algunas reflexiones acerca de lo que podría ser pensar y razonar sin especular. Es decir, si razonar sólo fuese deducir y abducir pero sin inducir.

Palabras clave: pensamiento, razonamiento, conjetura, especulación, búsqueda de especulaciones.

\section{Abstract}

Supported on a very simple mathematical model for Commonsense Reasoning, some reflections on what could be thinking and reasoning without speculating, are presented. That is, if reasoning only were deducing and abducing but without inducing.

Keywords: thinking, reasoning, conjecture, speculation, search of speculations.

\section{Introducción}

Los limites de mi lenguaje son los limites de mi mundo. Ludwig Wittgenstein

De Jenófanes de Colofón (580/570 AC - 475/466 AC) a Nicolás de Cusa (1401-1464) (Cfr. Popper (1963), Manzo (2014) y D’Amico (2018), la idea de que cuanto podemos conocer no son más que conjeturas fue permeando

Recibido: 10/12/2019. Aceptado: 18/02/2020. 
la interpretación del conocimiento humano. Mucho después, Karl R. Popper (1901-1994) en su libro Conjeturas y refutaciones. El crecimiento del conocimiento científico (Popper, 1963), reforzó lo expresado por Jenófanes quien, en uno de sus 'poemas filosóficos' había escrito 'cuanto podemos conocer son tejidos de suposiciones' y que el Cusano, en su libro De coniecturis, expresó como 'toda afirmación humana no es sino una conjetura'.

Sin embargo, en la obra de Nicolás de Cusa, esencialmente teológica (Cfr. Manzo (2014) y D’Amico (2018), la conjetura y la semejanza o analogía tienen un notable papel como el concepto de la docta ignorantia pone de relieve; un concepto éste no muy lejano al socrático 'Yo sólo sé que no sé nada' expuesto por Platón. Por su parte, Popper emplea la palabra 'conjetura' en el sentido de 'hipótesis', una identificación que, aunque acorde con la práctica científica, oculta gran parte del rico concepto de conjetura.

Ninguno de ellos, sin embargo, aporta una definición formal de conjetura ni, mucho menos, consideran el de especulación, un nuevo concepto que no apareció hasta el año 2000 (Trillas, S. Cubillo, E. Castiñeira, (2000)) y, entonces, limitado a los retículos con negación fuerte y única.

En este artículo se presenta un estudio formal del razonamiento, un modelo matemático muy general y simple del mismo (Trillas, (2018a)) que, englobando lo presentado en Trillas, S. Cubillo, E. Castiñeira, (2000) como un caso particular, tanto permite ver los conceptos de conjetura y de refutación con notable amplitud, como los diversos tipos de conjeturas que cabe considerar y algunas de las conclusiones que cabe extraer. En particular, permite ver bajo qué condiciones las consecuencias y las hipótesis son conjeturas, de lo cual lo primero ya había sido planteado, aunque no resuelto, por William Whewell (1794-1866) y Bernard Bosanquet (1848-1921), como se observa en los libros Novum Organum Renovatum y Theory of Scientific Method (Cfr. Whewell (1858), (1989)) del primero y Logic or The Morphology of Knowledge (volúmenes I y II) (Cfr. Bosanquet (1911)), del segundo. Libros en los cuales la inducción juega un rol esencial, por más que tampoco aporten una definición formal de conjetura ni, mucho menos, consideren las especulaciones. En la línea del juego de la inducción, también cabe señalar a John Stuart Mill con su libro A System of Logic, Ratiocinative and Inductive (Stuart Mill, (1872)), más bien favorable a la deducción y a quien Whewell discute duramente en (Cfr., Whewell, 1989).

Siguiendo la regla metodológica de Ockham-Menger (Menger, (1974), Trillas, (2019)), es decir, sin presuponer más axiomas que los necesarios ni menos que los suficientes, se mostrará cuándo la deducción es un caso particular de conjeturar así cómo y además, se concretará a qué cabe llamar 
inducción, a preguntarse si ésta es reducible a la deducción y cómo y cuándo lo es. En cierta forma, es una pregunta complementaria a la de Whewell, la cual podría enunciarse como 'la deducción debe ser un tipo de inducción'; en realidad, lo que se mostrará insinúa la disolución del concepto de inducción al reducirse ésta, en algún caso, a concatenar los dos tipos posibles de deducción.

Realmente, el último objetivo de este artículo es intentar desvelar el papel esencial que en el pensamiento, que lo contiene todo, juega el concepto de especulación (Trillas, (2017), Trillas, (2018a)) para poder razonar fructíferamente y más allá de lo conocido. Como el de especulación es un concepto nuevo, previamente hay que recorrer un camino formal para llegar a él y así poder intentar el desvele de aquel rol; plantearlo, por lo menos.

\section{Los conceptos formales de refutación y conjetura}

Conocer está, al fin, basado en reconocer. Ludwig Wittgenstein

Razonar, gracias al pensamiento, no es sólo inferir una conclusión q de una premisa $\mathrm{p}$, es mucho más. Si denotamos por el símbolo $<$ la relación de inferencia, aquella que se establece entre dos enunciados p y q de poderse afirmar el enunciado condicional 'Si p, entonces q', simbólicamente $\mathrm{p}<\mathrm{q}$, se dispone de una relación binaria que, al estar en el lenguaje y no entenderse siempre de la misma manera, consideraremos 'primitiva'. Entonces y denotando al enunciado no-q por q', la relación doblemente negativa 'No es: Si p, entonces no-q', simbólicamente [No ( $\left.p<q^{\prime}\right) \Leftrightarrow p</$ '], define, de afirmarla, que q es una conjetura de p (Trillas, (2017), (2018a), (2019)).

Definición 1. Dada p tal que $\mathrm{p}<\mathrm{p}$ y $\mathrm{p}</ \mathrm{p}$ ', q es una conjetura de $\mathrm{p}$ si $\mathrm{p}</ \mathrm{q}$ '.

Así, sabiendo $\mathrm{p}=$ Lucirá el Sol toda la mañana, cabe conjeturar $\mathrm{q}=$ No lloverá en toda la mañana, puesto que no cabe afirmar 'Si p, entonces lloverá', no es $\mathrm{p}<\mathrm{q}$ ', suponiendo que es q' = no (no lloverá en toda la mañana) = lloverá en algún momento de la mañana. Análogamente, al comprar un boleto del sorteo de Navidad, afirmar 'Este boleto tendrá el primer premio', no es sino una conjetura ya que cuanto sabemos acerca del sorteo no permite afirmar que no lo tendrá; jugamos a la lotería conjeturalmente, al igual que muchos de nuestros pensamientos habituales nos llevan a conjeturas con las que abordamos aspectos importantes de nuestras vidas. Por ejemplo, la decisión de Mengana de casarse con Mengano se basa en conjeturar que el matrimonio saldrá bien. 
Nótese que si en el caso de la mañana soleada, la conjetura está apoyada por la experiencia ('siempre es así'), ello no es el caso ni en el de la lotería, ni en el del matrimonio. Si de la primera conjetura cabe decir que está basada en la experiencia de lo sucedido anteriormente, de la segunda que lo está en el conocimiento del 'sistema' y de la tercera que lo está en una expectativa personal. En el razonamiento ordinario, con el cual el común de la gente toma muchas decisiones de su vida, las conjeturas se basan en motivos muy diversos y de los cuales se prescinde en lo que seguirá.

2.1. Se partirá de la relación primitiva <, llamada de inferencia y de la cual sólo se supondrá que es reflexiva, es decir, verifica $\mathrm{p}<\mathrm{p}$ para todo enunciado $\mathrm{p}$ y que, de ser la premisa de un razonamiento, su punto de partida, también verifica $\mathrm{p}</ \mathrm{p}$ ', es decir, no es auto-contradictoria. Nótese que, por ejemplo, si $\mathrm{p}=$ Lucirá el sol toda la mañana, no es $\mathrm{p}</$ p' = No lucirá el sol en toda la mañana; tal premisa p no es auto-contradictoria.

No cabe suponer que $<$ sea simétrica, [p $<\mathrm{q} \& \mathrm{q}<\mathrm{p}$ ], puesto que no es siempre el caso de que 'Si p, entonces q' pueda afirmarse conjuntamente con 'Si q, entonces p'; es el caso, por ejemplo, de 'Si llueve, entonces hay nubes' $\mathrm{y}$ 'Si hay nubes, entonces llueve' que no cabe afirmar. Cuando coinciden $\mathrm{p}<$ q y q $<$ p los enunciados son inferencialmente equivalentes y se escribirá p q para denotarlo. Dados dos enunciados p y q puede que simultáneamente no sean $\mathrm{p}<\mathrm{q}$ ni $\mathrm{q}<\mathrm{p}$, caso en el que los dos enunciados son incomparables $\mathrm{u}$ ortogonales respecto de la relación $<$ de inferencia y lo cual se denota por $\mathrm{p} \diamond \mathrm{q}$. Es el caso, por ejemplo, de $\mathrm{p}=$ Juan es rico y $\mathrm{q}=$ Juan es fotogénico.

Tampoco cabe afirmar que para cada terna de enunciados (p, q, r) y supuesto que sea $\mathrm{p}<\mathrm{q}$ y $\mathrm{q}<\mathrm{r}$, también sea $\mathrm{p}<\mathrm{r}$; es decir, que la relación $<$ sea transitiva. Por ejemplo, si con $\mathrm{p}=$ Llueve y estoy en la calle sin paraguas, $\mathrm{q}$ $=$ Me mojo y $\mathrm{r}=\mathrm{Al}$ llegar a casa me sube la fiebre hasta 38 grados, puede aceptarse que sea $\mathrm{p}<\mathrm{q}$ y $\mathrm{q}<\mathrm{r}$, pero no que sea $\mathrm{p}<\mathrm{r}$ necesariamente. Si la reflexividad de $<$ es universal, válida para todo enunciado, equivalencia, ortogonalidad y transitividad son, sin embargo, propiedades locales, de pares de enunciados las dos primeras y de ternas de ellos la tercera.

Inferir desde p para tener una conclusión q, puede hacerse hacia adelante, $\mathrm{p}<\mathrm{q}$, o hacia atrás, $\mathrm{q}<\mathrm{p}$. En el primer caso se habla de deducir q y en el segundo de abducir q. Lo deducido suele llamarse una consecuencia de la premisa y lo abducido una hipótesis de la premisa.

Definición 2. Una negación (') es toda aplicación tal que a cada enunciado $\mathrm{p}$ le hace corresponder otro p', único y de manera que: $\mathrm{p}<\mathrm{q}=>\mathrm{q}^{\prime}<\mathrm{p}$ ' (la negación invierte la relación $<$ de inferencia).

Teorema 1. Si $<$ es transitiva para la terna (p, q', p'), p < q implica $\mathrm{p}</ \mathrm{q}^{\prime}$. 
En efecto, si siendo $\mathrm{p}<\mathrm{q}$ fuese $\mathrm{p}<\mathrm{q}$, como sea que de la primera se sigue q' $<$ p', la transitividad de la terna (p, q', p') implicaría el absurdo $\mathrm{p}<$ p'. Por tanto, necesariamente es $\mathrm{p}</ \mathrm{q}$ '.

En esas condiciones, toda consecuencia es una conjetura, deducir es conjeturar y es obvio que el recíproco no vale; de llover puede afirmarse que hay nubes, pero éstas no aseguran ncesariamente que llueva.

Teorema 2 . Si $<$ es transitiva para la terna $(\mathrm{p}, \mathrm{h}, \mathrm{h}$ '), $\mathrm{h}</$ h' y $\mathrm{h}<\mathrm{p}$, implican $\mathrm{p}</$ h'.

En efecto, siendo $h<p$, si fuese $p<$ h' resultaría $h<h$ '. Por tanto, necesariamente debe ser $\mathrm{p}</ \mathrm{h}$ '. Bajo transitividad, toda hipótesis no autocontradictoria es una conjetura; en esas condiciones, abducir es un caso particular de conjeturar.

Definición 3. Un enunciado e que sea una cconjetura es una especulación desde la premisa $\mathrm{p}$, si $\mathrm{p} \diamond \mathrm{e}$.

Las especulaciones son conjeturas, tales que $\mathrm{p}</$ e' y son de dos tipos: Especulaciones débiles si verifican $\mathrm{p}</ \mathrm{e}$ ' en la forma $\mathrm{e}$ ' $<\mathrm{p}$, y especulaciones fuertes si verifican $\mathrm{p}</ \mathrm{e}$ ' en la forma $\mathrm{p} \triangleright \mathrm{e}^{\prime}$.

Las especulaciones débiles son aquellas cuya negación, de no ser autocontradictoria, es una hipótesis de la premisa y las fuertes aquellas cuya negación es ortogonal a la premisa.

Definición 4. Un enunciado $r$ es una refutación de la premisa $\mathrm{p}$, si verifica $\mathrm{p}<\mathrm{r}$ '. Refutar es obtener enunciados cuya negación sea deducible de la premisa; también se dice que r contradice o es contradictorio con p.

Por tanto, dada una premisa $\mathrm{p}$ no existen más que conjeturas o refutaciones de $\mathrm{p}$. Una refutación $\mathrm{r}, \mathrm{p}<\mathrm{r}$, no puede ser una conjetura de $\mathrm{p}, \mathrm{p}</ \mathrm{r}$; salvo si verifica r' $<p$, caso en que sería r' $\sim$ p y se caería en el absurdo $p$ $<$ p y $\mathrm{p}</$ p. Nótese que, en principio, la contradicción no es una relación simétrica: Puede ser $\mathrm{p}<\mathrm{r}$ ' sin que sea $\mathrm{r}<\mathrm{p}$ '.

Teorema 3. Si es $\mathrm{p}<\mathrm{r}^{\prime}$ y $\mathrm{r}<\left(\mathrm{r}^{\prime}\right)^{\prime}$, de ser $<$ transitiva para la terna $\left(\mathrm{r},\left(\mathrm{r}^{\prime}\right)\right.$, p'), entonces también es $r^{\prime}<p$.

En efecto, como de $\mathrm{p}<\mathrm{r}^{\prime}$ sigue $\left(\mathrm{r}^{\prime}\right)^{\prime}<\mathrm{p}^{\prime}$ y siendo $\mathrm{r}<\left(\mathrm{r}^{\prime}\right)^{\prime}$, la transitividad de la terna $\left(r,\left(r^{\prime}\right) ', p^{\prime}\right)$ lleva a $r<p^{\prime}$. Bajo esas condiciones, la contradicción es simétrica.

Definición 5. La negación puede ser: Débil en p, si $\mathrm{p}<\left(\mathrm{p}^{\prime}\right)^{\prime}$ e intuicionista en $\mathrm{p}$, si ( $\left.\mathrm{p}^{\prime}\right)^{\prime}<\mathrm{p}$. Si es a la vez débil e intuicionista, es $\mathrm{p} \sim\left(\mathrm{p}^{\prime}\right)^{\prime}$ y se llama fuerte en $\mathrm{p}$. Si no es ni débil ni intuicionista, es $\mathrm{p} \diamond\left(\mathrm{p}^{\prime}\right)^{\prime}$ y se llama salvaje en $\mathrm{p}$.

Nótese que, si la negación es débil o fuerte en r, el anterior teorema 3 permite afirmar que si $r$ refuta a $p$, entonces $p$ refuta a $r$. 
Teorema 4. Una negación fuerte es, bajo equivalencia, biyectiva.

En efecto, la definición 2 ya asegura que es inyectiva. Además es exhaustiva, puesto que dado cualquier p existe el único p' $=\mathrm{q}$, tal que q' $=\left(\mathrm{p}^{\prime}\right)^{\prime} \sim \mathrm{p}$.

Por lo tanto, el anterior teorema 3 puede reescribirse como: Una condición suficiente para que la contradicción sea simétrica, es que < sea transitiva y la negación débil o fuerte para los elementos involucrados. Así, no cabe asegurar tal simetría si no hay transitividad o la negación es bien puramente intuicionista, o bien es salvaje.

Definición 6. Una conjunción es toda operación $(\cdot)$ entre enunciados, tal que: $\mathrm{p} \cdot \mathrm{q}<\mathrm{p} \mathrm{y} \mathrm{p} \cdot \mathrm{q}<\mathrm{q}$, para cualesquiera enunciados $\mathrm{p}$ y $\mathrm{q}$.

Definición 7. Una disyunción es toda operación $(+)$ entre enunciados, tal que: $\mathrm{p}<\mathrm{p}+\mathrm{q}$ y $\mathrm{q}<\mathrm{p}+\mathrm{q}$.

Teorema 5. Cualquiera que sea la conjunción $(\cdot)$, p una premisa y e una especulación de $\mathrm{p}$, si $\mathrm{p}$ - e no es auto-contradictorio, es una hipótesis para p.

En efecto, es $\mathrm{p} \cdot \mathrm{e}<\mathrm{p}$. Nótese que no es necesario que e sea una especulación para que $\mathrm{p} \cdot \mathrm{e}$ sea una hipótesis, sucede lo mismo con cualquier enunciado e; sin embargo y siendo p la premisa de un razonamiento, parece más adecuado que, de buscarse una hipótesis, e mantenga alguna relación con p.

Teorema 6. Cualquiera que sea la disyunción (+), si p es una premisa y e una especulación de $\mathrm{p}, \mathrm{p}+\mathrm{e}$ es una consecuencia de $\mathrm{p}$.

En efecto, es $\mathrm{p}<\mathrm{p}+\mathrm{e}$. Nótese que no es necesario que e sea una especulación para que $\mathrm{p}+\mathrm{e}$ sea una consecuencia; cabe un comentario análogo al del anterior teorema 5: lo mismo sucede con cualquier enunciado e.

Teorema 7. Cualquiera que sea la conjunción $(\cdot)$, si < es transitiva y p es una premisa en la cual la negación es débil o fuerte y e una especulación de $\mathrm{p}$, entonces $\mathrm{p} \cdot \mathrm{e}$ es una refutación de $\mathrm{p}$.

En efecto, de p' e < p' sigue (p')' $<\left(\mathrm{p}^{\prime} \cdot \mathrm{e}\right)^{\prime}$ que, al ser $\mathrm{p}<\left(\mathrm{p}^{\prime}\right)^{\prime}$ lleva hasta $\mathrm{p}<\left(\mathrm{p}^{\prime} \cdot \mathrm{e}\right)^{\prime}$. Tampoco aquí es necesario que e sea una especulación. Nótese que la debilidad de la negación en $\mathrm{p}$ ya implica que, sin siquiera transitividad, p' sea una refutación de p.

Teorema 8. Dada una conjunción $(\cdot)$, si la negación es intuicionista o fuerte, la operación definida por $\mathrm{p} V \mathrm{q}=\left(\mathrm{p}^{\prime} \cdot \mathrm{q}\right)$ ' es una disyunción. Se llama la 'dual' de $(\cdot)$.

En efecto, de p' $<\mathrm{p}^{\prime} \cdot \mathrm{q}^{\prime}$ sigue $\left(\mathrm{p}^{\prime} \cdot \mathrm{q}^{\prime}\right)^{\prime}<\left(\mathrm{p}^{\prime}\right)^{\prime} \mathrm{y}$, siendo $\left(\mathrm{p}^{\prime}\right)^{\prime}<\mathrm{p}$, sigue $\left(\mathrm{p}^{\prime}\right.$ $\left.\cdot \mathrm{q}^{\prime}\right)^{\prime}=\mathrm{p} V \mathrm{q}<\mathrm{p}$. Análogamente, de $\mathrm{q}^{\prime}<\mathrm{p}^{\prime} \cdot \mathrm{q}^{\prime}$ se sigue $\mathrm{p} V \mathrm{q}<\mathrm{q}$.

Teorema 9. Dada una disyunción (+), si la negación es débil o fuerte, la operación definida por $\mathrm{p} \Lambda \mathrm{q}=\left(\mathrm{p}^{\prime}+\mathrm{q}\right.$ ')' es una conjunción. Se llama la 'dual' de (+). 
En efecto, de $\mathrm{p}^{\prime}+\mathrm{q}^{\prime}<\mathrm{p}^{\prime}$ sigue $\left(\mathrm{p}^{\prime}\right)^{\prime}<\left(\mathrm{p}^{\prime}+\mathrm{q}^{\prime}\right)^{\prime}=\mathrm{p} \Lambda \mathrm{q}$ y siendo $\mathrm{p}<\left(\mathrm{p}^{\prime}\right)^{\prime}$, sigue $\mathrm{p}<\mathrm{p} \Lambda \mathrm{q}$. Análogamente, de $\mathrm{p}^{\prime}+\mathrm{q}^{\prime}<\mathrm{q}^{\prime}$ se sigue $\mathrm{q}<\mathrm{p} \Lambda \mathrm{q}$.

Nótese que: a) Si la negación es fuerte, valen simultáneamente los teoremas 7,8 y 9. b) Si $(\cdot)$ (respectivamente, $(+)$ ) es conmutativa, también lo es $\mathrm{V}$ (respectivamente, $\Lambda)$. c) Si n es neutro para $(\cdot)$, n' lo es, bajo equivalencia, para $\left.V: n^{\prime} V q=\left(\left(n^{\prime}\right)^{\prime} \cdot q^{\prime}\right)^{\prime} \sim\left(n \cdot q^{\prime}\right)^{\prime}=\left(q^{\prime}\right)^{\prime} \sim q . d\right)$ Si m es neutro para $(+)$, m' lo es, bajo equivalencia, para $\Lambda: \mathrm{m}^{\prime} \Lambda \mathrm{q}=\left(\left(\mathrm{m}^{\prime}\right)^{\prime}+\mathrm{q}^{\prime}\right)^{\prime} \sim\left(\mathrm{m}+\mathrm{q}^{\prime}\right)^{\prime}=\left(\mathrm{q}^{\prime}\right)^{\prime}$ $\sim \mathrm{q}$.

Teorema 10. Cualesquiera que sean la conjunción, la disyunción y la negación, si < es transitiva, valen: 1) El 'principio' de No-contradicción, $\mathrm{p}$ $\cdot \mathrm{p}^{\prime}<\left(\mathrm{p} \cdot \mathrm{p}^{\prime}\right)^{\prime}$; para cualquier $\mathrm{p}$, es $\mathrm{p} \cdot \mathrm{p}^{\prime}$ auto-contradictorio, imposible; 2$)$ El 'principio' de Tercero Excluido, $\left(\mathrm{p}+\mathrm{p}^{\prime}\right)^{\prime}<\left(\left(\mathrm{p}+\mathrm{p}^{\prime}\right)^{\prime}\right)$ '; para cualquier $\mathrm{p}$, es $(p+p$ ')' auto-contradictorio, imposible.

En efecto, de $\mathrm{p} \cdot \mathrm{p}^{\prime}<\mathrm{p}$ sigue $\mathrm{p}^{\prime}<\left(\mathrm{p} \cdot \mathrm{p}^{\prime}\right)^{\prime} \mathrm{y}$, como también es $\mathrm{p} \cdot \mathrm{p}^{\prime}<\mathrm{p}^{\prime}$, por la transitividad sigue $\mathrm{p} \cdot \mathrm{p}^{\prime}<\left(\mathrm{p} \cdot \mathrm{p}^{\prime}\right)^{\prime}$. Análogamente, de $\mathrm{p}<\mathrm{p}+\mathrm{p}^{\prime}$ sigue $\left(\mathrm{p}+\mathrm{p}^{\prime}\right)^{\prime}<\mathrm{p}^{\prime} \mathrm{y}$, siendo también p' $<\mathrm{p}+\mathrm{p}^{\prime}$, sigue, a su vez, $\left(\mathrm{p}+\mathrm{p}^{\prime}\right)^{\prime}<\mathrm{p}+\mathrm{p}^{\prime}$ y de ello $\left(p+p^{\prime}\right)^{\prime}<\left(\left(p+p^{\prime}\right)^{\prime}\right)^{\prime}$.

Definición 8. Una operación ( $\&$ ), (respectivamente, $\mathbb{a}$ ) entre enunciados genera la relación $<$ sí y sólo sí es $\mathrm{p}<\mathrm{q} \Leftrightarrow \mathrm{p}=\mathrm{p} \& \mathrm{q}$, o respectivamente $\mathrm{p}$ $<\mathrm{q} \Leftrightarrow \mathrm{q}=\mathrm{p}$ a $\mathrm{q}$.

Teorema 11. Si $<$ tiene mínimo $\mathrm{n}$ y $(\&)$ genera $<$ en la forma $\mathrm{p}<\mathrm{q} \Leftrightarrow \mathrm{p}$ $=\mathrm{p} \& \mathrm{q}$, entonces $\mathrm{n}$ es absorbente para $(\&)$ por la izquierda.

En efecto, para cualquier $\mathrm{t}$, es $\mathrm{n}<\mathrm{t} \Leftrightarrow \mathrm{n} \& \mathrm{t}=\mathrm{n}$. Si $(\&)$ fuese conmutativa, n sería absorbente por la derecha y por la izquierda.

Nótese que la misma prueba muestra que si $(\&)$ genera $<$ y tiene absorbente por la izquierda, éste es mínimo para <. Es decir, en tales condiciones, mínimo y absorbente por la izquierda coinciden.

Teorema 12. En las condiciones del teorema 11, si < tiene máximo m, éste es neutro por la derecha.

En efecto, para cualquier $\mathrm{p}$ es $\mathrm{p}<\mathrm{n} \Leftrightarrow \mathrm{p}=\mathrm{p} \& \mathrm{n}$. De ser $(\cdot)$ conmutativa, $\mathrm{m}$ sería neutro por la derecha y por la izquierda.

Teorema 13. Si $<$ tiene máximo $\mathrm{m}$ y $(\mathrm{a})$ genera $<$ en la forma $\mathrm{p}<\mathrm{q} \Leftrightarrow \mathrm{q}$ $=\mathrm{p}$ a $\mathrm{q}$, entonces $\mathrm{m}$ es neutro para $(\mathrm{Q})$ por la derecha.

En efecto, para todo $t$ es $t<m \Leftrightarrow t a m=t$. $S i(a)$ fuese conmutativa, $m$ sería neutro por la derecha y por la izquierda; si lo fuese (\&), n sería absorbente por la derecha y por la izquierda.

Nótese que la misma prueba muestra que si (a) genera $<$ y tiene neutro por la derecha, éste es máximo para <. Es decir, en tales condiciones, máximo y neutro por la derecha coinciden. 
Teorema 14. En las condiciones del teorema 13, si < tiene mínimo, éste es neutro por la izquierda para (a).

En efecto, para todo $r$ es $n<r \Leftrightarrow r=n$ a $r$. De ser (a) conmutativa, el mínimo sería neutro por la izquierda y por la derecha.

$\mathrm{Si}<$ tiene un máximo 1 y $(\&)$ es una conjunción, llamando 'complemento relativo' de q en $\mathrm{p}$ (denotado p-q) a p \& q', resulta $1-\mathrm{r}=\mathrm{r}$ ', la negación de cualquier $r$ es su complemento relativo al máximo que, por el teorema 12 , es neutro por la derecha para la conjunción (\&).

\section{La búsqueda de conjeturas}

Una proposición sin prueba no pertenece a la misma categoría que una proposición con prueba. Ludwig Wittgenstein

No se razona siempre mediante uno o dos pasos como sucede con $\mathrm{p}<$ $\mathrm{q}$, con $\mathrm{h}<\mathrm{p} \mathrm{y} \mathrm{h}<$ h', así como con e $\nabla$ p y e' $<$ p, para afirmar, respectivamente, que q es una consecuencia, h una hipótesis y e una especulación débil. Usualmente, el razonamiento se produce mediante cadenas o caminos de inferencia hasta llegar a una conclusión satisfactoria para el problema en cuestión; como es, por ejemplo, la cadena [p $<\mathrm{r}, \mathrm{r}<\mathrm{s}, \mathrm{s}<\mathrm{t}, \mathrm{t}<\mathrm{q}]$ para llegar desde $\mathrm{p}$ a $\mathrm{q}$.

Sin embargo, a menos que < sea transitiva, la cadena anterior no permite asegurar que q sea una consecuencia de $\mathrm{p}$, que sea $\mathrm{p}<\mathrm{q}$, ni muestra cómo, en cada eslabón de ella, se encuentra el consecuente correspondiente a cada antecedente. ¿Cómo puede encontrarse el consecuente de un antecedente dado?

Frecuentemente se encuentra mediante el proceso consistente en partir del primer antecedente, $\mathrm{p}$, especular $\mathrm{e}_{1}$ desde él y tomar $\mathrm{r}=\mathrm{p}+\mathrm{e}_{1}$ como consecuente; hecho esto en cada eslabón, resulta finalmente $\mathrm{q}=\left(\left(\left(\left(\mathrm{p}+\mathrm{e}_{1}\right)+\right.\right.\right.$ $\left.\left.\left.\mathrm{e}_{2}\right)+\mathrm{e}_{3}\right)+\mathrm{e}_{4}\right)$, puesto que entre p y q median cuatro eslabones.

Para obtener una hipótesis $\mathrm{h}$, la cadena podrá ser del tipo: $\mathrm{p}>\mathrm{r}, \mathrm{r}>\mathrm{s}$, $\mathrm{s}>\mathrm{t}, \mathrm{t}>\mathrm{h}($ con $\mathrm{u}>\mathrm{w} \Leftrightarrow \mathrm{w}<\mathrm{u}$ ) y que, sin transitividad, tampoco permite asegurar que h sea una hipótesis de $\mathrm{p}$. En ese caso, puede procederse análogamente con $\mathrm{r}=\mathrm{p} \cdot \mathrm{e}_{1}$ y llegar hasta $\mathrm{h}=\left(\left(\left(\left(\mathrm{p} \cdot \mathrm{e}_{1}\right) \cdot \mathrm{e}_{2}\right) \cdot \mathrm{e}_{3}\right) \cdot \mathrm{e}_{4}\right)$.

Nótese que sólo si las operaciones $+\mathrm{y}(\cdot)$ son asociativas puede escribirse $\mathrm{q}=\mathrm{p}+\left(\mathrm{e}_{1}+\ldots+\mathrm{e}_{4}\right) \mathrm{yh}=\mathrm{p} \cdot\left(\mathrm{e}_{1} \cdot \ldots \cdot \mathrm{e}_{4}\right)$. En todo caso y bajo transitividad, es $\mathrm{p}<\mathrm{q}$ y $\mathrm{h}<\mathrm{p}$, ya que, por ejemplo, es $\mathrm{q}>\ldots>\left(\left(\left(\mathrm{p}+\mathrm{e}_{1}\right)+\mathrm{e}_{2}\right)+\mathrm{e}_{3}\right)>\ldots>$ p y, análogamente, es $\mathrm{h}<\ldots<\left(\left(\mathrm{p} \cdot \mathrm{e}_{1}\right) \cdot \mathrm{e}_{2}\right)<\ldots<\mathrm{p}$. 
Por lo que respecta a las especulaciones, obviamente no pueden encontrarse mediante sólo caminos hacia adelante como las consecuencias, ni sólo mediante caminos hacia atrás como las hipótesis. Inducir es buscar especulaciones.

Por otra parte, si < es transitiva, no habrá más conjeturas que las consecuencias, las hipótesis y las especulaciones, con lo cual existe la posibilidad de emplear cadenas mixtas, quebradas, hacia adelante y hacia atrás, o al revés y que, no pudiendo dar lugar a consecuencias o hipótesis, sólo podrán dar lugar a elementos ortogonales, que serán especulaciones supuesto que valgan sus dos propiedades (Trillas $\&$ de Soto (p.p.)). Así y partiendo de p, $\mathrm{k}=\left(\mathrm{p} \cdot \mathrm{e}_{1}\right)+\mathrm{e}_{2}$ verifica $\mathrm{p} \cdot \mathrm{e}_{1}<\mathrm{p}$ y $\mathrm{p} \cdot \mathrm{e}_{1}<\left(\mathrm{p} \cdot \mathrm{e}_{1}\right)+\mathrm{e}_{2}=\mathrm{k}$, y permite llegar de $\mathrm{p}$ a k mediante la quebrada $\mathrm{p}>\mathrm{p} \cdot \mathrm{e}_{1}<\mathrm{k}$; faltará obviamente asegurarse que bien es $\mathrm{k}^{\prime}<\mathrm{p}$, o bien k' $\nabla \mathrm{p}$, para poder afirmar que k es una especulación. Sin embargo, conocidos la premisa p y la especulación e tales quebradas existen; por lo menos existe la $\mathrm{p}>\mathrm{p} \cdot \mathrm{e}<\mathrm{e}$. Naturalmente, que existan quebradas desde $\mathrm{p}$ no significa que con ellas siempre se encuentren especulaciones e, solamente significa que se llegará a elementos ortogonales a p.

Sin embargo, para encontrar todas las conclusiones posibles de un enunciado $\mathrm{p}$ en el caso particular de un álgebra de Boole finita y, por tanto, atómica, con un número finito de átomos, basta utilizar los teoremas 5 y 6 , con un enunciado e cualquiera. Por ejemplo, en el álgebra de Boole finita con los cinco átomos a, b, c, d, e y con los $2^{5}$ elementos que se obtienen añadiendo átomos por disyunción $(+)$, partiendo de $\mathrm{p}=\mathrm{a}+\mathrm{b}$ que, siendo $\mathrm{p}^{\prime}=\mathrm{c}+\mathrm{d}+\mathrm{e}$, no es auto-contradictorio, se obtienen las nueve consecuencias:

p, $q=a+b+c, q=a+b+d, q=a+b+e, q=a+b+c+d, q=a+b+$ $c+e, q=a+b+d+c, q=a+b+d+e, q=a+b+c+d+e$,

así como las dos hipótesis $\mathrm{h}_{1}=\mathrm{a} \mathrm{y}_{2}=\mathrm{b}$, ninguna auto-contradictoria al ser $h_{1}{ }^{\prime}=b+c+d+e y h_{2}{ }^{\prime}=a+c+d+e$.

En este caso, para hallar las especulaciones basta añadir y quitar, o quitar y añadir, átomos, para así disponer de quebradas, llegar a un $e$ y comprobar luego si verifica $e \diamond \mathrm{p}$ y bien $e^{\prime}<\mathrm{p}$, o bien $e^{\prime} \diamond \mathrm{p}$. Por ejemplo, pasando de a $+\mathrm{b}$, hasta $\mathrm{b}$ y luego hasta $\mathrm{b}+\mathrm{c}=e$; obviamente es $\mathrm{a}+\mathrm{b} \backslash \mathrm{b}+\mathrm{c}(\mathrm{p} \backslash e) \mathrm{y}, \mathrm{al}$ ser $e^{\prime}=\mathrm{a}+\mathrm{d}+\mathrm{e}$ también es $e^{\prime} \nabla \mathrm{p}$, es decir, $e$ es una especulación fuerte de p. Del mismo p se obtiene la especulación débil $e=\mathrm{c}+\mathrm{d}+\mathrm{e}$, puesto que es $\mathrm{p} \diamond e \mathrm{y} e^{\prime}=\mathrm{p}$. La primera especulación se obtiene con la quebrada $\mathrm{p}>\mathrm{b}<$ $e$ y la segunda con los pasos $\mathrm{p}, \mathrm{p}^{\prime}=e$. Es una vía para encontrar sistemáticamente todos los $e$ ortogonales a p y, luego, seleccionar entre ellos los que bien verifiquen $e^{\prime}<\mathrm{p}$, o bien verifiquen $e^{\prime} \nabla \mathrm{p}$, es decir, las especulaciones débiles y las fuertes, respectivamente. 
Nótese que $\mathrm{p}^{\prime}=\mathrm{c}+\mathrm{d}+\mathrm{e}$ y la especulación débil $e=\mathrm{c}+\mathrm{e}$, dan $\mathrm{p}^{\prime} \cdot e=$ $\mathrm{d}$, con el cual es $\left(\mathrm{p}^{\prime} \cdot e\right)^{\prime}=\mathrm{a}+\mathrm{b}+\mathrm{c}+$ e y $\mathrm{p}=\mathrm{a}+\mathrm{b}<\left(\mathrm{p}^{\prime} \cdot e\right)^{\prime}$, es decir y de acuerdo con el teorema $7, \mathrm{p}^{\prime} \cdot e=\mathrm{c}$ es una refutación de $\mathrm{p}$.

Debe remarcarse que en cualquier álgebra de Boole existe un único elemento auto-contradictorio, el mínimo 0 . En efecto, $\mathrm{p}<\mathrm{p}^{\prime}=>\mathrm{p} \cdot \mathrm{p}=\mathrm{p}<\mathrm{p}$. $\mathrm{p}^{\prime}=0$; el recíproco es también fácil, ya que al ser $0^{\prime}=1$ es $0<0$ '. Es $0^{\prime}=1$ puesto que $0<$ n para todo n, implica n' $<0$ ' para todo n' (teorema 4); con ello y además, $1^{\prime}=\left(0^{\prime}\right)^{\prime}=0$, por ser la negación fuerte. Basta que p no sea 0 para que no sea auto-contradictorio. Tales resultados valen igualmente para cualquier orto-retículo y álgebra de De Morgan; en esas estructuras las pruebas anteriores son las mismas.

Teorema 15. Si < es transitiva, las especulaciones débiles e de p se obtienen bien por deducción si la negación es débil en e, o bien por abducción si la negación es débil en $\mathrm{p}$ e intuicionista en e.

En efecto, la condición e' < p implica p' < (e')' y si la negación es intuicionista en e, (e')' <e, la transitividad lleva a p' < e; e se alcanza deductivamente a partir de la negación de la premisa. Si la negación es débil en $\mathrm{p}, \mathrm{p}<$ (p')', la transitividad lleva desde e'< p hasta e' $<\left(p^{\prime}\right)^{\prime}$, con lo cual es $\left(\left(p^{\prime}\right)^{\prime}\right)^{\prime}$ $<\left(\mathrm{e}^{\prime}\right)^{\prime}$ y, siendo (e')' < e, se llega a $\left(\left(\mathrm{p}^{\prime}\right)^{\prime}\right)^{\prime}<$ e.

En el ejemplo anterior, con $\mathrm{p}=\mathrm{a}+\mathrm{b}, \mathrm{p}^{\prime}=\mathrm{c}+\mathrm{d}+\mathrm{e}$ coincide con la especulación $e$. En el caso en que la negación es intuicionista o fuerte en e, la expresión resultante p' $<$ e, indica que e es una consecuencia de p' $=$ no-p.

Nótese que el teorema es válido cuando la negación es fuerte, en cuyo caso es $\left(\left(p^{\prime}\right)^{\prime}\right)$ ' $\sim$ p', y deja de valer si la negación es bien salvaje, o bien si es estrictamente débil en e y estrictamente intuicionista en p.

De cuanto se ha mostrado hasta aquí, del ejemplo finito y del teorema anterior, cabe preguntarse si, por lo menos en los orto-retículos y las álgebras de De Morgan y, en particular, las álgebras de Boole, donde la inducción se limita a las especulaciones fuertes, inducir no es sino una mezcla de deducir y abducir. ¿Cabe hacer la hipótesis de que ello es general? Refutarlo requiere un contraejemplo, aceptarlo requiere probarlo; lo único probado hasta ahora es su validez en las álgebras de Boole finitas (Trillas \& de Soto (p.p))

Como una curiosidad final para esta sección,

Teorema 16.

a) Si la negación es débil en $\left(\mathrm{r}^{\prime}\right)^{\prime}=\mathrm{r}^{\prime}$, es $\left(\mathrm{r}^{\prime}\right)^{\prime}<\left(\left(\left(\mathrm{r}^{\prime}\right)^{\prime}\right)^{\prime}\right)^{\prime} \Leftrightarrow \mathrm{r}^{\prime}<\mathrm{r}^{\mathrm{iv}}$.

b) Si la negación es intuicionista en $r^{\prime \prime}$, es $\left(\left(\left(r^{\prime}\right)^{\prime}\right)^{\prime}\right)^{\prime}<\left(r^{\prime}\right)^{\prime} \Leftrightarrow r^{\text {iv }}<r^{\prime \prime}$.

c) Si la negación es fuerte en $r$, es $r \sim r$ ” y r" $\sim r^{\text {iv }}$. 
En efecto, el primer caso es inmediato sin más que abreviar ( $\mathrm{r}^{\prime}$ )' por $\mathrm{s}$, ya que es $\mathrm{s}<\mathrm{s}$ ". El segundo lo es, con la misma abreviación, al ser $\mathrm{s}$ " $<\mathrm{s}$. En cuanto al tercero, es inmediato al ser $\mathrm{s} \sim \mathrm{s}$ ".

Análogamente se sigue $\mathrm{r}^{\mathrm{iv}}<\mathrm{r}^{\mathrm{vi}} \mathrm{y}$, si la negación es fuerte $\mathrm{y}<$ transitiva es $\mathrm{r} \sim \mathrm{r}^{\prime \prime} \sim \mathrm{r}^{\mathrm{iv}} \sim \mathrm{r}^{\mathrm{vi}} \sim \ldots$ que, empezando con r', lleva también a r' $\sim \mathrm{r}^{\prime \prime} \sim \mathrm{r}^{\mathrm{v}}$ $\sim \mathrm{r}^{\mathrm{vii}} \sim$..., cuando la negación es fuerte.

Nótese, como consecuencia de lo último y en el caso considerado por el Teorema 15, que si la negación es débil o fuerte en p, entonces la conclusión $\left(\left(\left(p^{\prime}\right)^{\prime}\right)^{\prime}<\right.$ e puede identificarse con p' < e, al igual que sucede si la negación es intuicionista o fuerte.

\section{Reflexiones adicionales}

Una proposición muestra lo que dice. Una tautología y una contradicción muestran que nada dicen. Ludwig Wittgenstein

Tras las definiciones y teoremas anteriores, se intentarán extraer algunas reflexiones adicionales.

4.1. La primera de ellas es que el marco matemático presentado es muy general, abarca mucho del razonamiento ordinario y permite, cuando ello convenga, añadir más definiciones llevando a resultados conocidos en las estructuras lógico-matemáticas usualmente empleadas en el análisis de tipos particulares de razonamiento (E. Trillas, S. Cubillo, E. Castiñeira (2000), Trillas (2017)). Podría decirse que las pocas propiedades que definen a $<$, $\left({ }^{\circ}\right),(\cdot)$ y $(+)$, con las cuales han aparecido los anteriores dieciséis teoremas como 'hechos formales', permiten mucho juego cuando el razonamiento se concreta; podría decirse que aquellas son las leyes formales básicas del razonamiento ordinario o de sentido común.

En cualquier caso, tales leyes valen en los retículos con negación fuerte como los orto-retículos, que son muy empleados en la representación matemática de partes del razonamiento como es, por ejemplo, el caso del razonamiento de la física cuántica (E. Trillas, S. Cubillo, E. Castiñeira (2000)) en el que se han empleado retículos orto-modulares y también en el del razonamiento impreciso con álgebras de conjuntos borrosos (fuzzy sets, en inglés) (Trillas (2017)) que, de ser un retículo, son una álgebra de De Morgan (Trillas (2017)) y que, de forma usual, gozan de todas aquellas pocas propiedades aún cuando no se trate de las llamadas álgebras estándar de fuzzy sets (Trillas (2017), Zadeh, (2014), Trillas, (2018a)). 
Por ejemplo, en el caso del razonamiento con propiedades nítidas, disponiendo potencialmente de cuanta información previa sea menester y sin incertidumbre, la estructura de álgebra de Boole se acepta como indiscutible (Trillas, García-Honrado, (2013)). Cuando las propiedades son precisas pero existe incertidumbre de tipo aleatorio, la adición al álgebra de Boole de una probabilidad, apoyada en los datos de tipo estadístico disponibles, es empleada con éxito en muchas ocasiones; pero, si las propiedades exhibidas son imprecisas o si la incertidumbre no es de tipo aleatorio, entonces puede ser interesante el uso de modelos distintos al de la probabilidad y como son, por ejemplo, las funciones de credibilidad o las medidas de posibilidad y probabilidad borrosa de Zadeh (Trillas (2017)).

$¿$ Qué leyes adicionales se requieren, por ejemplo, para poder contar con la llamada propiedad del 'reparto perfecto' $p=p \cdot q+p \cdot q$ ', para enunciados cualesquiera p y q? Se trata de una propiedad que, típica de las álgebras de Boole (Trillas, García-Honrado, (2013)), antecede a la propiedad aditiva de una medida de probabilidad.

Una prueba del reparto perfecto en las álgebras de Boole es la siguiente: $\mathrm{p}=\mathrm{p} \cdot 1=\mathrm{p} \cdot\left(\mathrm{q}+\mathrm{q}^{\prime}\right)=\mathrm{p} \cdot \mathrm{q}+\mathrm{p} \cdot \mathrm{q}^{\prime}$, que pone de manifiesto que se requieren: 1) La existencia de elemento neutro, 1, para la conjunción y que, de acuerdo con los teoremas 11 y 12 es, a la vez, absorbente para la disyunción $(+)$ y el máximo respecto del orden reticular del álgebra de Boole, $\mathrm{p}<\mathrm{q} \Leftrightarrow \mathrm{p}$ $=\mathrm{p} \cdot \mathrm{q} \Leftrightarrow \mathrm{q}=\mathrm{p}+\mathrm{q}$, generado por ambas operaciones $(\cdot)$ y $(+)$. 2) La validez de la ley distributiva $\mathrm{p} \cdot(\mathrm{q}+\mathrm{r})=\mathrm{p} \cdot \mathrm{q}+\mathrm{p} \cdot \mathrm{r}$.

La del reparto perfecto es una propiedad mostrando que todo $\mathrm{p}$ se reparte perfectamente entre q y q', cualquiera que sea q, y puesto que es $(\mathrm{p} \cdot \mathrm{q})$. $\left(\mathrm{p} \cdot \mathrm{q}^{\prime}\right)=(\mathrm{p} \cdot \mathrm{p}) \cdot\left(\mathrm{q} \cdot \mathrm{q}^{\prime}\right)=\mathrm{p} \cdot 0=0$, en virtud de las muchas propiedades de las álgebras de Boole y como son la asociatividad, la conmutatividad y la idempotencia de la conjunción.

El reparto perfecto está, a su vez, en el fondo de la equivalencia entre contradicción e incompatibilidad, entre $\mathrm{p}<\mathrm{q}$ ' y $\mathrm{p} \cdot \mathrm{q}=0$, respectivamente y para cuya formulación se requiere la existencia del mínimo del álgebra, 0 , el cual, también de acuerdo con los teoremas 11 y 12, es neutro para la disyunción y absorbente para la conjunción. Para verlo, basta el siguiente cálculo, $\mathrm{p}=\mathrm{p} \cdot \mathrm{q}+\mathrm{p} \cdot \mathrm{q}^{\prime}=0+\mathrm{p} \cdot \mathrm{q}^{\prime}=\mathrm{p} \cdot \mathrm{q}^{\prime} \Leftrightarrow \mathrm{p}<\mathrm{q}^{\prime}$, indicando que la incompatibilidad implica la contradicción; para el recíproco, de $\mathrm{p}<\mathrm{q}$, sigue, $\mathrm{p} \cdot \mathrm{q}<\mathrm{q}^{\prime} \cdot \mathrm{q}=0=\mathrm{p} \cdot \mathrm{q}=0$, que maneja la ley, en general no supuesta, de monotonía $[\mathrm{p}<\mathrm{q}=>\mathrm{p} \cdot \mathrm{r}<\mathrm{q} \cdot \mathrm{r}$, para cualquier $\mathrm{r}$ ], así como la ley q' $\cdot \mathrm{q}=$ $\mathrm{q} \cdot \mathrm{q}^{\prime}=0$ equivalente, a su vez, a q $\cdot \mathrm{q}^{\prime}<\left(\mathrm{q} \cdot \mathrm{q}^{\prime}\right)^{\prime}$, la de No-contradicción anterior. 
En las álgebras de Boole, en el cálculo proposicional clásico (Trillas, García-Honrado, (2013)), no se distinguen contradicción e incompatibilidad como sucede de ordinario; los principios de No-contradicción y Tercero Excluido se presentan en forma de los axiomas $\mathrm{p} \cdot \mathrm{p}^{\prime}=0$ y $\mathrm{p}+\mathrm{p}^{\prime}=1$, en ellas equivalentes a lo presentado en el teorema 10. En las álgebras de Boole 'p y no-p' se considera imposible cuando es un enunciado equivalente al mínimo 0 del retículo, en tanto que 'no (p o no-p))' se considera imposible o 'p o no-p' totalmente posible, cuando 'p o no-p' es equivalente al máximo 1 del retículo.

Un mínimo y un máximo que no se han supuesto en general puesto que, en primer lugar, su existencia es dudosa en los razonamientos habituales y, en segundo lugar y como muestran los teoremas 11 y 12, fuerzan propiedades algebraicas que, en principio, no cabe suponer siempre en el lenguaje ordinario con el que se expresa el razonamiento de sentido común.

En las álgebras de Boole, así como en todos los orto-retículos y las álgebras de De Morgan, vale la ley de dualidad $(\mathrm{p}+\mathrm{q})^{\prime}=\mathrm{p} \cdot \mathrm{q}$ ', con la cual es $\mathrm{p} \cdot \mathrm{q}=\left(\mathrm{p}^{\prime}+\mathrm{q}\right)^{\prime}$ '; es decir, + es la disyunción $(\mathrm{V})$ asociada por el teorema 7 a la conjunción $(\cdot)$ y ésta es la conjunción $(\Lambda)$ del teorema 8 . Se trata de operaciones duales y, por tanto, dada una de ellas y la negación, se obtiene la otra; algo que no es siempre universal en el razonamiento de sentido común, no lo es en el lenguaje ordinario donde algunas veces ambas operaciones son independientes.

4.2. Teorema 17. Dos consecuencias cualesquiera de la misma premisa $\mathrm{p}$, no pueden ser, bajo transitividad, contradictorias.

En efecto, si son $\mathrm{p}<\mathrm{q}$ y $\mathrm{p}<\mathrm{r}$, de ser $\mathrm{q}<$ r' por ejemplo, de $\mathrm{p}<\mathrm{q}$ seguiría $\mathrm{p}<\mathrm{r}$ ' y de $\mathrm{p}<\mathrm{r}$ seguiría $\mathrm{r}^{\prime}<\mathrm{p}$ ' con lo cual y finalmente se llegaría al absurdo $\mathrm{p}<\mathrm{p}$ '. En particular, si la premisa no lo es, ninguna consecuencia puede ser auto-contradictoria; la deducción con transitividad nunca lleva a la contradicción.

Por lo que respecta a las hipótesis, no cabe garantizar que si es $\mathrm{h}<\mathrm{p}$ nunca sea $h$ auto-contradictoria y es por ello que se exige que sea $h</$ h' para considerarla una hipótesis. Tampoco cabe garantizar que dos hipótesis distintas de p no sean nunca contradictorias, de lo cual hay ejemplos reales.

En cuanto a las especulaciones,

Teorema 18. Las especulaciones débiles no pueden ser, bajo transitividad, auto-contradictorias.

En efecto, de e' $<$ p, si fuese e $<$ e' seguiría, bajo transitividad, e $<$ p, lo cual es absurdo al ser e $\downarrow$ p; por tanto, siempre es e $</$ e'. 
Por su parte y de nuevo, no cabe garantizar que una especulación fuerte no sea auto-contradictoria e, igual que con las hipótesis, es algo a comprobar en cada caso. Lo mismo sucede con las refutaciones; en general y aunque no sean auto-contradictorias, nada cabe asegurar respecto a la posible contradicción de dos de ellas.

4.3. La información p que permite efectuar un razonamiento no se mantiene siempre fija, varía, aparece nueva información $\mathrm{p}^{*}$ que, de no contradecir a p, debe agregársele $\mathrm{y}$, así, volver a empezar pero de la nueva conjunción $\mathrm{p} \cdot \mathrm{p} *$. Con ello, es natural preguntarse si lo que antes de $\mathrm{p} *$ se consideró una conclusión puede mantenerse como tal; es el llamado problema de la monotonía del razonamiento (Trillas, 2018a).

Teorema 19. Conjeturar es, bajo transitividad, anti-monótono, es decir, al aumentar las premisas, no aumentan las conjeturas.

En efecto, si $\mathrm{p} \cdot \mathrm{p}^{*}</$ q', de ser $\mathrm{p}<\mathrm{q}^{\prime} \mathrm{y}$ de $\mathrm{p} \cdot \mathrm{p}^{*}<\mathrm{p}$, seguiría $\mathrm{p} \cdot \mathrm{p}^{*}<$ q'; por tanto, es $\mathrm{p}</$ q'. Toda 'nueva' conjetura ya lo era antes; su número no aumenta.

Teorema 20. Refutar es, bajo transitividad, monótono, es decir, al aumentar las premisas, no disminuyen las refutaciones.

En efecto, si $\mathrm{p}<\mathrm{r}^{\prime}$ y como es $\mathrm{p} \cdot \mathrm{p}^{*}<\mathrm{p}$, sigue $\mathrm{p} \cdot \mathrm{p}^{*}<\mathrm{r}^{\prime}$. Toda antigua refutación sigue siendo una nueva refutación; su número no disminuye.

Teorema 21. Deducir es, bajo transitividad, monótono.

En efecto, si $\mathrm{p}<\mathrm{q}$, de $\mathrm{p} \cdot \mathrm{p}^{*}<\mathrm{p}$, sigue $\mathrm{p} \cdot \mathrm{p}^{*}<\mathrm{q}$.

Teorema 22. Abducir es, bajo transitividad, anti-monótono.

En efecto, si $\mathrm{h}<\mathrm{p} \cdot \mathrm{p}^{*}$, de $\mathrm{p} \cdot \mathrm{p}^{*}<\mathrm{p}$, sigue $\mathrm{h}<\mathrm{p}$.

Por cuanto respecta a las especulaciones, no cabe asegurar, en general, ni que sean monótonas ni anti-monótonas; en principio y simplemente sólo cabe decir de ellas que son no-monótonas, que su comportamiento con el aumento de las premisas no es, en general, predecible.

Lo más que puede decirse de las especulaciones es que las débiles son, en algunos casos, anti-monótonas. Es así, por ejemplo, cuando siendo e $\diamond$ $\left(\mathrm{p} \cdot \mathrm{p}^{*}\right)$ y también e $\nabla \mathrm{p}$, es e' $<\mathrm{p} \cdot \mathrm{p}^{*}$ ya que de $\mathrm{p} \cdot \mathrm{p}^{*}<\mathrm{p}$ sigue $\mathrm{e}^{\prime}<\mathrm{p}$ bajo transitividad. Realmente, en tales casos no hay 'nuevas' especulaciones.

Nótese que la monotonía muestra que, de ordinario y con el aumento de las premisas, el número de las refutaciones y el de las consecuencias crece, suele haber más, en tanto que la anti-monotonía muestra que, de ordinario, el número de las conjeturas y las hipótesis decrece, suele haber menos.

Si la monotonía muestra que siempre cabe encontrar más refutaciones y más conclusiones deductivas, que la deducción hacía adelante no tiene fin, la anti-monotonía muestra que a medida que se sabe más de algo, menos va 
quedando por conjeturar y abducir, que puede llegar un momento en el cual ya no quede nada por explicar desde una cierta premisa, que la deducción hacia atrás tiene fin.

Ello es, no obstante, irreal; cuando se llega a una tal situación suele aparecer un nuevo punto de vista desde el cual el problema adquiere una nueva perspectiva que dirige hacia nuevas preguntas, preguntas en otra dirección u otra intención. Si toda respuesta tiene fecha, aun desconocida, de caducidad y, también tal vez de respuesta, el objeto de la pregunta evoluciona y ofrece nuevas preguntas. El mundo del pensamiento es, por emplear el título de la autobiografía de Karl R. Popper, una búsqueda sin fin. Además, los teoremas 18 a 20 anteriores requieren la transitividad de $<$, la cual no puede suponerse siempre y cuando se manejen especulaciones en particular.

4.4. La transitividad de la relación < juega un papel esencial para obtener la mayoría de los teoremas que se han presentado, pero no siempre puede suponerse que $<$ sea transitiva. Por ejemplo, si $\mathrm{p}=$ paseo por la calle, $\mathrm{q}=$ un niño tropieza conmigo, $\mathrm{r}=$ el niño cae, cabe suponer $\mathrm{p}<\mathrm{q}$ y $\mathrm{q}<\mathrm{r}$, pero es dudoso que necesariamente quepa suponer $\mathrm{p}<\mathrm{r}$. Sin $\mathrm{q}$ el último condicional ni siquiera es sensato. Por ello, se han buscado definiciones alternativas a la transitividad como es, por ejemplo, [p $<\mathrm{q}, \mathrm{q}<\mathrm{r}=>\mathrm{p} \cdot \mathrm{q}<\mathrm{r}$ ], con la cual el anterior ejemplo cobra sentido y que, de ser $<$ generada por $(\cdot)$ en la forma, $\mathrm{r}<\mathrm{s} \Leftrightarrow \mathrm{r}=\mathrm{r} \cdot \mathrm{s}$, puede llevar $\mathrm{p} \cdot \mathrm{q}<\mathrm{r}$ a $\mathrm{p}<\mathrm{r}$, si es $\mathrm{p}=\mathrm{p} \cdot \mathrm{q}$; puede reducirse a la transitividad. Es una forma de transitividad que podría llamarse 'débil'.

En el caso particular que < sea generada por una operación * y en la forma $\mathrm{t}<\mathrm{s} \Leftrightarrow \mathrm{t}=\mathrm{t} * \mathrm{~s}$, entonces [p $<\mathrm{q} \& \mathrm{q}<\mathrm{r}$ ], equivalen a [ $\mathrm{p}=\mathrm{p} * \mathrm{q} \&$ $\mathrm{q}=\mathrm{q} * \mathrm{r})=>(\mathrm{p}=(\mathrm{p} * \mathrm{q}) * \mathrm{r})]$, que, de ser $*$ conmutativa implica $\mathrm{p}=\mathrm{p} *(\mathrm{q}$ $* \mathrm{r}) \Leftrightarrow \mathrm{p}<\mathrm{q} * \mathrm{r}$ y que, de ser $*$ una conjunción, al verificar $\mathrm{q} * \mathrm{r}<\mathrm{r}$, lleva a $\mathrm{p}<\mathrm{r}$, a la ley transitiva Una conjunción conmutativa genera, por tanto, una relación $<$ transitiva. También debe notarse que la ley reflexiva $\mathrm{p}<\mathrm{p}$ exige que sea $\mathrm{p}=\mathrm{p} * \mathrm{p}$, que la conjunción (*) sea idempotente. Análogamente puede procederse con una operación generadora en la forma alternativa $\mathrm{t}<\mathrm{s} \Leftrightarrow \mathrm{s}=\mathrm{t} * \mathrm{~s}$, cuando $(*)$ sea una disyunción conmutativa que se verá forzada a ser idempotente.

La validez de los resultados obtenidos en las secciones segunda y tercera de este artículo dependen, casi todos, de la transitividad de $<$ y del carácter de la negación ('), así como, algunos pocos, de propiedades de la conjunción $(\cdot)$ y de la disyunción $(+)$. Son condiciones que no pueden suponerse siempre en el razonamiento ordinario y que, cuando se dan, se está en un razonamiento particular, con más formalismo; cabría decir, por ejemplo, que bajo transitividad, la deducción y la abducción son 'formales', propias 
de una cierta representación matemática admisible y en la cual no puede suceder que una consecuencia sea auto-contradictoria, ni que dos consecuencias se contradigan.

Cabe, por tanto, distinguir entre inferencia ordinaria con, a lo más, transitividad local, e inferencia formal con transitividad universal; distinguir así la deducción ordinaria, la de la persona corriente, de la deducción formal de un matemático (Trillas, (2017), (2018a), (2019)).

Sin transitividad, por ejemplo, pueden existir consecuencias e hipótesis que no sean conjeturas; es un mundo por explorar y en el cual parece que establecer nuevas y más débiles formas de transitividad, para poder avanzar en lo inferido o para preservar que deducir es conjeturar, es relevante. La transitividad simplifica muchísimo el mundo de la conjetura.

Nótese, por ejemplo, que si es $\mathrm{p}<\mathrm{q}$, supuesto que fuese $\mathrm{p}<\mathrm{q}$ ' y como de la primera sigue q' < p', con la anterior transitividad débil sería $\mathrm{p} \cdot \mathrm{q}$ ' $<$ p' que, para llevar al absurdo $\mathrm{p}<\mathrm{p}$ ' y forzando $\mathrm{p}</ \mathrm{q}$ ', puede requerir $\mathrm{p} \sim \mathrm{p}$ - q', una condición que no es independiente de los particulares p y q de los que se trate. Son, como se ha dicho, preguntas a explorar.

4.5. Con frecuencia, tanto los caminos deductivos hacía adelante o hacía atrás y con una relación < transitiva, pueden programarse informáticamente, son algoritmos computables; sin embargo, no es obvio que ello pueda hacerse para hallar especulaciones y con los que hemos llamado caminos mixtos o quebrados, en los cuales no se sabe a priori cuando retroceder o avanzar. Es algo que obliga a conocer más que la premisa p de partida; debe 'conocerse un entorno' de la misma para poder decidir, por lo menos, si y cuándo se comienza a avanzar o a retroceder (Trillas \& de Soto (p.p))

Es un problema que tiene una larga historia comenzando por el libro De Coniecturis, de Nicolás de Cusa y citado al comienzo; en él, el Cusano, sugiere emplear la máquina de Ramon Llull, el Ars (Trillas, 2019), para encontrar conjeturas. Una máquina inventada para deducir y con la que Llull buscaba, por ejemplo, todos los términos medios de silogismos permitiendo llegar de una premisa dada a una consecuencia prefijada; algo parecido a lo establecido en el teorema 6. Posiblemente, algunos de los algoritmos de la Inteligencia Artificial que emplean las llamadas búsquedas heurísticas, encuentren muchas veces conjeturas con cierta probabilidad de validez que, no obstante y soslayando a veces problemas de alta complejidad computacional, pueden arrojar conclusiones 'peligrosas' (Trillas, 2018a) al no poderse explicar claramente cómo se ha obtenido una conclusión.

Sin embargo, el problema de maquinizar la búsqueda de especulaciones está, realmente, abierto y puede tropezar fácilmente con una tal explosión 
combinatoria que lo haga computacionalmente intratable; basta pensar en el caso de un álgebra de Boole finita con un elevadísimo número de átomos y en el cual, dada la premisa p, el número de elementos e tales que sea e $\diamond$ $\mathrm{p}$ puede ser enorme.

\section{Conclusión}

La filosofía no es una teoría sino una actividad. Ludwig Wittgenstein

5.1. Con todo, el problema del 'razonamiento mecanizado' debe distinguirse del razonamiento humano el cual, continuamente asistido por el pensamiento (que lo contiene como una variedad del 'dirigido a objetivos'), cuenta con otros recursos provenientes de la analogía con situaciones anteriores y de destellos creativos, realmente especulativos y tanto favorecidos por aquellas analogías, como por nueva información adquirida a través de la observación o la experimentación (Trillas (2020)).

En el caso humano, la conjetura no sólo viene auxiliada por el conocimiento sino también por la experiencia del sujeto (Trillas, (2018a)), una experiencia usualmente múltiple y no sólo proveniente de cuanto sepa del problema en cuestión. Una experiencia que puede ser, también, limitante para conjeturar en la buena dirección y de lo cual ejemplos como el de 'querer ver' la circunferencia como la 'trayectoria perfecta' de los planetas, ponen de manifiesto. Así se creyó hasta que, mejores observaciones, hicieron ver que se requería otra conjetura distinta y surgieron las leyes de Kepler.

Quienes se dedican a la investigación científica tienden a preguntarse sobre los fenómenos sean físicos o virtuales, e intentando que sus preguntas sean buenas, es decir, lleven a conjeturas facilitando conclusiones comprobables y fértiles; algo para lo cual su 'ojo clínico'(Trillas, (2017), (2018b)), adquirido a través del estudio, la experiencia, el ensayo y error, la corrección de errores anteriores, etc., juega un gran papel. Un papel que no excluye que alguien con menos experiencia, pero necesariamente muy estudioso, no acierte con una buena pregunta sobre el conocimiento aportado por otros y a través de su crítica, haciendo una atrevida especulación y, con ella, llegar a una refutación, a una consecuencia, o a una hipótesis extraordinariamente fértil y, tal vez, como muestran los anteriores teoremas 5, 6 y 7 . Kepler lo hizo con las observaciones de Tycho Brahe, mucho mejores que las de Copérnico. 
5.2. Al aumentar el conocimiento siempre podrán obtenerse más refutaciones y más consecuencias, así como menos conjeturas e hipótesis, pero con las especulaciones no cabe, en general, esperar lo mismo ya que carecen de ley de crecimiento o de decrecimiento, su no-monotonía las sitúa a un lado del razonamiento y en su borde interior con el pensamiento dirigido.

Las refutaciones permiten reenfocar las situaciones, los problemas; las consecuencias advertir nuevas aplicaciones y tanto teóricas como prácticas; las hipótesis permiten encontrar explicaciones cada vez más finas, dependientes de menos cosas y abriendo el campo a nuevas propiedades, pero las especulaciones, tal vez basadas en una refutación, abren el campo a lo realmente nuevo, a la 'creación'. Las especulaciones pueden ser el 'chispazo' llevando a ver algo de otra manera y, a partir de ello, a nuevas refutaciones, nuevas consecuencias y nuevas hipótesis. Sin el roce entre pensamiento libre, refutación y deducción, no habría especulación; no se iría más lejos.

Una de las vías para 'ir más lejos' es la síntesis entre un enunciado y su opuesto facilitando una conjetura, la sintesis dialéctica que, proveniente de Hegel, es tan cara a los pensadores marxistas y, en realidad, no muy alejada de la coincidentia oppositorum empleada por Nicolás de Cusa en sus argumentaciones teológicas (Manzo, (2014)). Una vía que recientemente se ha conseguido explicar (Trillas, I. García-Honrado, (2019)) empleando cuanto aparece en las anteriores secciones 2,3 y 4, así como ejemplificándola mediante conjuntos borrosos y que ya ha sido aplicado a un problema computacional (Mordeson, Mathew (p.p.))

5.3. Aceptando que la verdad de los enunciados se expresa por medio de funciones $t$ con valores en el intervalo unidad $[0,1]$ y tales que (Cfr. Trillas(2018a)):

1) Si $\mathrm{p}<\mathrm{q}$, entonces $\mathrm{t}(\mathrm{p}) \leq \mathrm{t}(\mathrm{q}), 2) \mathrm{t}\left(\mathrm{p}^{\prime}\right)=1-\mathrm{t}(\mathrm{p})$,

es obvio que las consecuencias no disminuyen o aumentan el 'grado t' de verdad de la premisa, las hipótesis no lo aumentan o lo disminuyen, las refutaciones $\left(\mathrm{p}<\mathrm{r}^{\prime}=>\mathrm{t}(\mathrm{p})+\mathrm{t}(\mathrm{r}) \leq 1\right)$, si son completamente verdaderas hacen completamente falsa a la premisa, en tanto que si son falsas nada indican de la verdad de la misma.

En cuanto a las especulaciones sólo cabe decir algo respecto de las débiles, ya que $e^{\prime}<\mathrm{p}=>1 \leq \mathrm{t}(\mathrm{e})+\mathrm{t}(\mathrm{p})$ : Una especulación débil completamente falsa lo es, necesariamente, de una premisa completamente verdadera, pero una completamente verdadera no implica nada sobre la verdad de la premisa y, por su parte, si la premisa es verdadera las especulaciones débiles tanto pueden ser verdaderas como falsas, en tanto que si la premisa es falsa las especulaciones débiles serán siempre verdaderas. En cuanto a las 
especulaciones fuertes, las expresiones e $\diamond \mathrm{p}$ y e' $\diamond \mathrm{p}$ no permiten relacionar $\mathrm{t}(\mathrm{e})$ con $\mathrm{t}(\mathrm{p})$, se trata de valores independientes; nada cabe decir de ellos. Las especulaciones fuertes son salvajes y tanto desde el punto de vista de la inferencia, de la auto-contradicción, de la monotonía, como de la verdad.

En todo caso, lo visto sobre los tipos de conclusiones parece advertir que sólo los valores 0 y 1 , correspondientes a completamente falso y completamente verdadero, no son realistas. Por ejemplo, de ser $t(p)=0.8$, una premisa $\mathrm{p}$ muy verdadera, entonces las consecuencias q tendrán un grado de verdaderas entre 0.8 y 1 , las hipótesis h lo tendrán entre 0 y 0.8 , las refutaciones $r$ entre 0 y 0.2 , y las especulaciones débiles e entre 0.2 y 1 . En este ejemplo, el mayor rango de posible variación corresponde a hipótesis y especulaciones débiles, el menor a consecuencias y refutaciones.

De ser $\mathrm{t}(\mathrm{p})=0.2$ una premisa p poco verdadera, aquellos rangos serían, respectivamente, entre 0.2 y 1 , entre 0 y 0.2 , entre 0 y 0.8 , y entre 0.8 y 1 ; con ello, los mayores rangos de variación corresponderían a consecuencias e hipótesis, en tanto que los menores a refutaciones y especulaciones débiles.

El grado de verdad de la premisa condiciona fuertemente la variabilidad de los grados de consecuencias, hipótesis, refutaciones y especulaciones débiles, sin que nada quepa prever de las especulaciones fuertes. Valores de $\mathrm{t}$ intermedios entre 0 y 1 , permiten más consideraciones que sólo los dos 0 y 1 .

A este último respecto, puede ser ilustrativo referirse al ejemplo, en la sección 3, del álgebra de Boole con cinco átomos a, b, c, d, e. Supuestos los cinco con grado $1 / 5$ y supuesto que, por ejemplo, $\mathrm{t}(\mathrm{p})=\mathrm{t}(\mathrm{a}+\mathrm{b})=\mathrm{t}(\mathrm{a})+\mathrm{t}$ (b), que es un 'grado' de verdad, será $\mathrm{t}(\mathrm{p})=2 / 5$ y para la especulación débil $e=\mathrm{b}+\mathrm{c}+\mathrm{d}+\mathrm{e}$, será $\mathrm{t}(e)=4 / 5$, en tanto que para la especulación fuerte $e$ $=\mathrm{b}+\mathrm{c}$ será $2 / 5$. La primera se mantiene en el rango previsible $[3 / 5,1]$, pero la segunda está fuera del mismo.

5.4. Para finalizar, las especulaciones y esencialmente las nunca obtenibles de forma deductiva, las fuertes, son las que provocan el sorprendente fenómeno de 'encontrar lo nuevo' (Trillas, (2018a)); hacen que lo sorprendente del pensamiento sea su capacidad de explicación por medio de la refutación, la deducción y la abducción.

La especulación se sale de estas tres vías para, inmediatamente, volver a ellas; es el fuelle del razonamiento, zig-zageando en el pensamiento las especulaciones inducen lo nuevo y son, así, el germen de la creatividad. En cierta medida, ese zig-zagueo viene espoleado por la pasión que en la búsqueda ponga el pensador (Trillas, (2018a), (2018b). Las emociones humanas juegan ahí un notable papel; ayudan, como muestran múltiples ejemplos, a que el cerebro siga buscando, pensando en cierta línea, aun sin la consciencia del sujeto. 
Si el pensamiento libre puede considerarse espontáneo, generado por y en el cerebro con ayuda de los sentidos, el pensamiento dirigido a un objetivo se produce gracias a la voluntad del sujeto; es intencional y sólo considerar al razonamiento como deducción y abducción, sería incompleto. Las especulaciones fuertes son el caos que, en el pensamiento dirigido, permite el orden del razonamiento.

Sin poder especular y mayormente de forma fuerte, el razonamiento quedaría limitado a sólo avanzar y retroceder, a deducir y abducir; sería menos de lo mucho que es realmente, quedaría limitado por las dos fronteras marcadas por tales avances y retrocesos, sólo tendría dos dimensiones. Es el zig-zag de la especulación lo que añade al razonamiento el 'salto' a más dimensiones; dentro del pensamiento, lo lleva al más allá.

La especulación permite pensar aquello que se busca a partir del conocimiento inicial, la premisa, conjeturarlo antes de cualquier prueba y tanto si lo buscado es una consecuencia o una explicación, una hipótesis. Sin especular, razonar carecería de perspectiva, sería como un mundo plano habitado por seres planos. Se razona más allá de algo por cuanto se dispone, en el pensamiento dirigido y abierto, de algo poco regulado; de la especulación, en suma.

En resumen, sin la posibilidad de especular, el razonamiento se acercaría al actual de las máquinas, los ordenadores; es decir, sería más simple y regulado. Sería un razonamiento alejado de lo ortogonal, de lo que no es deductivamente comparable con lo conocido; seguramente carecería del chispazo creativo (Trillas, (2018a)). En realidad, sería razonamiento sin pensamiento.

\section{Agradecimientos}

1) A la memoria de Ludwig Wittgenstein (1889-1951), Karl Menger (1902-1985) y Lotfi A. Zadeh (1921-2017); sin las sugerencias recibidas de sus obras respectivas (Menger (1974), Zadeh (2014) y Wittgenstein (1973)), el autor nunca habría escrito cuanto le ha llevado a este artículo.

2) A la memoria de Javier Muguerza (1936-2019) quien, amablemente, intentó reforzar las tendencias filosóficas del autor.

3) A la revista Ágora y su Comité Editorial, con agradecimiento a su permanente gentileza con el autor. 


\section{Bibliografía}

Bosanquet, B. (1911), Logic or The Morphology of Knowledge (Vols. I y II). Clarendon Press.

D’Amico, C., (2018), 'Acerca del carácter irreductible de la mens humana en Nicolás de Cusa: cantidad y número'. Franciscanum, 169/IX: 87-107.

Manzo, S. (2014), 'Possibilitas-Materia'. Manuductiones (Eds. C. Rusconi y K. Reinhard): 191-209. Aschendorff Verlag.

Menger, K., (1974), Morality, Decision and Social Organization. Toward a Logic of Ethics. Reidel.

Mordeson, J. N. \& S. Mathew, 'Dialectic Synthesis. Application to Human Trafficking'. En curso de publicación en New Mathematics and Natural Computation.

Popper, K. R., (1963), Conjectures and Refutations. The Growth of Scientific Knowledge. Routledge \& Kegan Paul.

Stuart Mill, J., (1872), A System of Logic, Ratiocinative and Inductive. Longmans, Green, Reader, and Dyer.

Trillas, E. (2017), On the Logos. A Naïve View of Ordinary Reasoning and Fuzzy Logic. Springer.

Trillas, E. (2018a), El desafío de la creatividad. Eds. Universidade de Santiago de Compostela.

Trillas, E. (2018b), 'Reflexión sobre investigar, la lógica borrosa y sus aplicaciones'. Ágora, 17/2: 7-25.

Trillas, E. (2019), 'Entre Llull y Ockham, el razonamiento'. Revista Internacional d' Humanitats, 49: 57-68.

Trillas, E. (2020), Narrar, conjeturar y calcular. El pensamiento. Eds. UGR.

Trillas, S. Cubillo, E. Castiñeira, (2000), 'Conjectures in orthocomplemented lattices'. Artificial Intelligence, 117: 255-275.

Trillas, E. \& I. García-Honrado, (2013), ‘ ¿Hacia un replanteamiento del cálculo proposicional clásico?’. Ágora, 12/1:7-25.

Trillas, E. \& I. García-Honrado, (2019), 'A Reflection on the Dialectic Synthesis'. New Mathematics and Natural Computation, 15/1: 31-46.

Trillas, E. \& A. de Soto, 'Searching Speculations'. En curso de publicación en New Mathematics and Natural Computation.

Wittgenstein, L. (1973), Philosophical Investigations. $3^{\text {a }}$ Edición, Prentice Hall.

Whewell, W. (1858), Novum Organum Renovatum. John W. Parker \& Son. Whewell, W. (1989), Theory of Scientific Method. Hackett Publishing Company. 